Universität Berlin, der o. Professor der Geschichte an der Universität Czernowitz $\mathrm{Ka}$ indl als o. Professor für österreichische Geschichte an die Universität Graz, der a.o. Professor der Geschichte an der Universitüt Innsbruck Steinacker als o. Professor für österreichische Geschichte an die deutsche Universität in Prag, der Privatdozent in Marburg Wolzendorff als a.o. Professor für Staats- und Verwaltungsrecht nach Königsberg i/Pr., der o. Professor für neuere Literaturgeschichte in Basel Unger an die Universität Halle, der o. Professor des deutschen und Kirchenrechts in Halle Fehr in gleicher Eigenschaft an die Universität Heidelberg, der o. Professor des deutschen und Kirchenrechts an der Universität Freiburg i/Br. A. Schultze in gleicher Eigenschaft an die Unive rsität Leipzig, der o. Professor des deutschen und Kirchenrechts an der Universität Giefen Hübner in gleicher Eigenschaft nach Halle, der a.o. Professor Zinkernagel in Tübingen als o. Professor für deutsche Philologie nach Basel, der a.o. Professor an der Universität Berlin v. Schwerin als o. Professor für deutsches Recht und Kirchenrecht an die Universität Strafaburg i/F., der o. Professor der Geschichte in Innsbruck Erben in gleicher Eigenschaft nach Graz, der o. Professor des deutschen Rechts an der Universität Göttingen $K$. Beyerle in gleicher Eigenschaft an die.Universität Bonn.

Befördert wurden: Der Privatdozent Frings in Bonn zum a.o. Professor für deutsche Philologie und Niederländisch daselbst, der a.o. Professor der Geschichte in Breslau Ziekursch zum o. Professor ebenda, der a.o. Professor in Graz v. Srbik zum o. Professor für neuere Geschichte und Wirtschaftsgeschichte daselbst, der Titularextraordinarius Goldmann in Wien zum etatsmäfigen a.o. Professor für deutsche Rechtsgeschichte und Rechtsaltertümer daselbst, der Privatdozent in Bern Mutzner zum a.o. Professor für deutsche und schweizerische Rechtsgeschichte ebenda.

Es habilitierten sich: In Wien Otto Stowasser für historische Hilfswissenschaften, in Berlin Walther Schubring für indogermanische Philologie, in Leipzig Heinrich Goessel für indogermanische Sprachwissenschaft, in München Alexander v. Müller für Geschichte.

\title{
Bericht der akademischen Kommission für das Wörterbuch der deutschen Rechtssprache
}

\author{
fur das Jahr 1916. \\ Von Hrn. R o ethe.
}

Eine Sitzung der akademischen Kommission hat auch im vertlossenen Jahre nicht stattgefunden. Ebensowenig konnte eine neve lieferung des Rechtswörterbuchs ausgegeben werden. Kriegsverwendung der Mitarbeiter und Druckschwierigkeiten trafen da zusammen. 
Die Kommission erkennt mit lebhaftem Danke an, daf ihr wiederum ein sehr erwünschter Geldzuschuf aus den Mitteln der SavignyStiftung bewilligt wurde.

Als der wissenschaftliche Leiter des Rechtswörterbuchs, Hr. Rich. Schröder in Heidelberg, um Weihnachten in gewohnter Weise gebeten wurde, den Jahresbericht abzustatten, lag er bereits an schwerer Krankheit darnieder. Seitdem hat ihn, an 3. Januar 1917, der Tod dahingerafft. So wurde das Rechtswörterbuch, bald nachdem ihm Hein rich Brunners wache Fïrsorge entzogen ward, von einem zweiten nicht minder schweren Verluste betroffen. Es ist hier nicht der Platz, Richard Schröders menschliche und wissenschaftliche Persönlichkeit zu würdigen. Aber die freudige Hingebung, nit der er seine unverwüstliche Kraft in den Dienst des Rechtswörterbuchs gestellt hatte, dem sein Herz und seine Gedanken treu blieben bis zuletzt, wird ihm von der Akademie nicht vergessen werden. Er stand schon in vorgerückten Jahren, als er sich an das groke Werk machte. Aber der glückliche, zuversichtliche Optimismus, mit dem er sich an die gewaltige Aufgabe wagte, kannte keinen $Z_{w}$ eifel und gab ihm die nie versagende Elastizität des Geistes und Willens, die ihn alle Schwierigkeiten heiter überwinden liek. Wie er mit ungetrübter Siegesgewifheit den Gefahren und Schwankungen des Krieges sichern Herzens zuschaute, so leitete ihn auch bei seiner Arbeit am Deutschen Rechtswörterbuch ein frohgemutes Zutrauen zum guten Erfolg, das er auf alle seine Freunde und Mitarbeiter ausstrahlte: wie oft haben wir uns bei den erquicklichen Heidelberger Kommissionssitzungen an diesem herzhaften Vertrauen erbaut! Hat er die Vollendung nicht erleben dürfen, so ist es ihm doch beschieden gewesen, wenigstens die ersten Hefte des geliebten, treu gehegten Werkes im Druck abgeschlossen zu erblicken. Brunner und er haben als die eigentlichen Väter des Deutschen Rechtswörterbuchs zu gelten: ihr Name ist mit seiner Geschichte wurzelhaft verwachsen.

An Stelle der Geschiedenen berichtet $\mathrm{Hr}$. Eberhard Freiherr von Kü n ₹berg, der, soweit sein Kriegshilfsdienst ihm dies gestattet, zur Zeit die Geschäfte und Arbeiten des Rechtswörterbuchs weiterführt, über den Fortgang des Unternehmens das Folgende:

Bericht des Hrn. Eberhard Freiherrn von Künßberg.

Auch im verflossenen Jahre war die Arbeit am Rechtswörterbuche beeinträchtigt. Der Druck konnte noch nicht fortgesetzt werden. Doch schreiten die Ordnungsarbeiten vorwärts, und der-wissenschaftliche Auskunftverkehr konnte aufrechterhalten werden.

Verzeichnis der im Jahre 1916 ausgezogenen Quellen:

Das kaiserliche Buch des Markgrafen AlbrechtAchilles: Dr. H. Crebert, München.

Altbayrische Monatsschrift: Dr. Crebert.

B iblio th ek des literarischen Vereins, Bd. 35 : Admiral Bachem, Heidelberg. Köb el, Gerichtsordnung 1523: Dr. Crebert. 
Stadtrechte von La ufenburg und Mellingen. Aarau 1915: Prof. Dr. v. Künßberg.

La u remberg, Scherzgedichte: Dr. Crebert.

Lettscher Notariatbuch 1576 : Dr. Crebert.

Lindner, Urkundenwesen Karls IV.: Dr. Crebert.

Mannheimer Geschichtsblätter 1915: Geheimrat R. Schröder.

Mitteilungen des Vereins für anhaltische Geschichte 10: Dr. Crebert. Niederrheinische Annalen: Geheimrat R. Schröder.

Quellen zur Rechts- und Wirtschaftsgeschichte I. Trier: Geheimrat R. Schröder.

Schwäbisches Wörterbuch II.: Prof. Dr. v. Künßberg.

Scbwarzemberg, Teutsche Cicero: Dr. Crebert.

To eppen, Elbinger Antiquititen: Dr. Crebert.

Ulm, Gesetz und Ordnungen der Stadt: Dr. Crebert.

Wusterwitz, Märkische Chronik: Dr. Crebert.

Zeitschrift für Rechtsgeschichte 1914, 1915: Prof. Dr, v. Künßberg. 